\title{
Equivalence classes of permutations modulo descents and left-to-right maxima
}

\author{
JEAN-LUC BARIL \\ Université de Bourgogne, B.P. 47870 \\ LE2I UMR-CNRS 6306 \\ 21078 Dijon, France \\ e-mail: barjl@u-bourgogne.fr \\ and \\ Armen Petrossian \\ Université de Bourgogne, B.P. 47870 \\ LE2I UMR-CNRS 6306 \\ 21078 Dijon, France \\ email: armen.petrossian@u-bourgogne.fr
}

(Received: July 8, 2014, and in revised form March 23, 2015)

\begin{abstract}
In a recent paper [2, the authors provide enumerating results for equivalence classes of permutations modulo excedances. In this paper we investigate two other equivalence relations based on descents and left-to-right maxima. Enumerating results are presented for permutations, involutions, derangements, cycles and permutations avoiding one pattern of length three.
\end{abstract}

Mathematics Subject Classification(2010). 05A05.

Keywords: permutation, equivalence class, descent, left-to-right maximum, pattern, Bell number, Motzkin number, Catalan number, Fine number.

\section{Introduction and notations}

Let $S_{n}$ be the set of length- $n$ permutations, i.e., all one-to-one correspondences from $[n]=\{1,2, \ldots, n\}$ into itself. The one-line notation of a permutation $\pi \in S_{n}$ is $\pi_{1} \pi_{2} \cdots \pi_{n}$ where $\pi_{i}=\pi(i)$ for $i \in[n]$. The graphical representation of $\pi \in S_{n}$ is the set of points in the plane at coordinates $\left(i, \pi_{i}\right)$ for $i \in[n]$. A cycle in $S_{n}$ is an $n$-length permutation $\pi$ such that there exist some indices $i_{1}, i_{2}, \ldots, i_{n}$ with $\pi\left(i_{1}\right)=i_{2}, \pi\left(i_{2}\right)=i_{3}, \ldots, \pi\left(i_{n-1}\right)=i_{n}$ and $\pi\left(i_{n}\right)=i_{1}$. A cycle will also be denoted by its cyclic notation $\pi=\left\langle i_{1}, i_{2}, \ldots, i_{n}\right\rangle$. Let $C_{n} \subset S_{n}$ be the set of all cycles of length $n$. We denote by $I_{n}$ the set of involutions of length $n$, i.e., permutations $\pi$ such that $\pi^{2}=I d$ where $I d$ is the identity permutation.

Let $\pi$ be a permutation in $S_{n}$. A fixed point of $\pi$ is a position $i \in[n]$ where $\pi(i)=i$. The set of $n$-length permutations with no fixed points (called derangements) will be denoted $D_{n}$. An excedance of $\pi$ is a position $i \in[n-1]$, such that $\pi(i)>i$. The set of excedances of $\pi$ will be denoted $E(\pi)$. A descent of $\pi$ is a position $i \in[n-1]$, such that $\pi(i)>\pi(i+1)$. Let $D(\pi)$ be the set of descents in $\pi$, and $D D(\pi)$ be the set of pairs $(\pi(i), \pi(i+1))$ for $i \in D(\pi)$. By abuse of language, we also use the

DOI: 10.1515/puma-2015-0002 
term descent for such a pair. A left-to-right maximum is a position $i \in[n]$, such that $\pi(i)>\pi(j)$ for all $j<i$. The set of left-to-right maxima of $\pi$ will be denoted $L(\pi)$. For instance, if $\pi=14275386$ then $E(\pi)=\{2,4,7\}, D(\pi)=\{2,4,5,7\}, D D(\pi)=\{(4,2),(7,5),(5,3),(8,6)\}$ and $L(\pi)=\{1,2,4,7\}$.

In [2], the authors consider the equivalence relation on $S_{n}$ in which two permutations $\pi$ and $\sigma$ are equivalent if they coincide on their excedance sets, i.e., $E(\pi)=E(\sigma)$ and $\pi(i)=\sigma(i)$ for $i \in E(\pi)$. In this paper we investigate the counterpart of this equivalence relation for descents and left-to-right maxima. More precisely, we define the $\ell$-equivalence relation $\sim_{\ell}$ where $\pi \sim_{\ell} \sigma$ if and only if $\pi$ and $\sigma$ coincide on their left-to-right maximum sets, i.e., $L(\pi)=L(\sigma)$ and $\pi(i)=\sigma(i)$ for $i \in L(\pi)$. Also, we define the $d$-equivalence relation $\sim_{d}$ where two permutations $\pi$ and $\sigma$ are equivalent if $D D(\pi)=D D(\sigma)$. The motivation for studying this $d$-equivalence relation is that two permutations $\pi$ and $\sigma$ are equivalent under excedance ([2]) if and only if $\phi(\pi)$ and $\phi(\sigma)$ are $d$-equivalent, where $\phi$ is the Foata's first transformation [5] (see Theorem 6). All these definitions remain available for subsets of $S_{n}$. For instance, the permutation $32541 \in S_{5}$ is $\ell$-equivalent to $32514,31524,31542$ and $d$-equivalent to 54132 . The set of $\ell$-equivalence (resp. $d$-equivalence) classes in $S_{n}$ is denoted $S_{n}^{\sim_{\ell}}\left(\operatorname{resp} . S_{n}^{\sim_{d}}\right.$ ).

In this paper we propose to compute the number of $\ell$ - and $d$-equivalence classes for several subsets of permutations.

A permutation $\pi \in S_{n}$ avoids the pattern $\tau \in S_{k}$ if and only if there does not exist any sequence of indices $1 \leq i_{1}<i_{2}<\cdots<i_{k} \leq n$ such that $\pi\left(i_{1}\right) \pi\left(i_{2}\right) \ldots \pi\left(i_{k}\right)$ is order-isomorphic to $\tau$ (see [13, 14]). We denote by $S_{n}(\tau)$ the set of permutations of $S_{n}$ avoiding the pattern $\tau$. For example, if $\tau=123$ then $52143 \in S_{5}(\tau)$ while $21534 \notin S_{5}(\tau)$. Many classical sequences in combinatorics appear as the cardinality of pattern-avoiding permutation classes. A large number of these results were firstly obtained by West and Knuth [8, 12, 13, 14, 15, 16] (see books of Kitaev [7] and Mansour [11]).

In Section 2, we investigate the equivalence relation based on the set of left-to-right maxima. We enumerate $\ell$-equivalence classes for $S_{n}, C_{n}, I_{n}, D_{n}$ and several sets of pattern avoiding permutations. In Section 3, we study equivalence relation for descents and also provide enumerating results for some restricted sets of permutations. See Table 1, 2 and 3 for an overview of these results.

\section{Enumeration of classes under $\ell$-equivalence relation}

Throughout this section two permutations $\pi$ and $\sigma$ belong to a same class whenever they coincide on their sets of left-to-right maxima, i.e., $L(\pi)=L(\sigma)$ and $\pi(i)=\sigma(i)$ for $i \in L(\pi)$.

A Dyck path of semilength $n, n \geq 0$, will be a lattice path starting at $(0,0)$, ending at $(2 n, 0)$, and never going below the $x$-axis, consisting of up steps $U=(1,1)$ and down steps $D=(1,-1)$. Let $\mathcal{P}_{n}$ be the set of all Dyck paths of semilength $n$. A peak of height $h \geq 0$ in a Dyck path is a point of ordinate $h$ which is both at the end of an up step and at the beginning of a down step.

From a permutation $\pi \in S_{n}$, we consider the path on the graphical representation of $\pi$ with up and right steps along the edges of the squares that goes from the lower-left corner to the upper-right corner and leaving all the points $\left(i, \pi_{i}\right), i \in[n]$, to the right and remaining always as close to the diagonal $y=x$ as possible (the path can possibly reach the diagonal but never crosses it). Let us define the Dyck path of length $2 n$ (called Dyck path associated with $\pi$ ) obtained from this lattice path by reading an up-step $U$ every time the path moves up, and a down-step $D$ every time the path moves to the right. It is crucial to notice that only the points $\left(i, \pi_{i}\right)$ with $i \in L(\pi)$ involve in this construction. See Figure 1 for an illustration of this classical construction. 

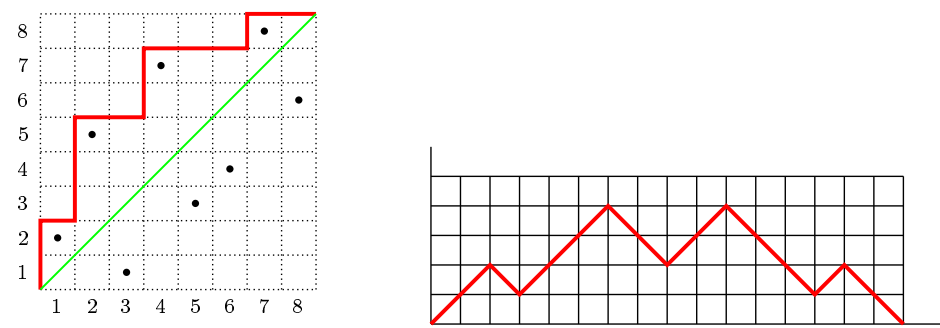

Figure 1: Permutation $\sigma=25173486$.

Using this construction, all permutations of a same class provide the same Dyck path. Moreover, any Dyck path in $\mathcal{P}_{n}$ can be obtained from a permutation in $S_{n}$. Indeed, we define the sequence $\ell=\ell_{1} \ell_{2} \ldots \ell_{r}$, (resp. $\left.k=k_{1} k_{2} \ldots k_{r}\right), r \geq 1$ where $\ell_{i}$ (resp. $k_{i}$ ) is the number (resp. the number plus one) of up steps $U$ (resp. down steps $D$ ) before the $i$-th peak. Since $P$ is a Dyck path, we have $k_{i} \leq \ell_{i}$ for $i \leq r$. So, we define the permutation $\pi=\ell_{1} A_{1} \ell_{2} A_{2} \ldots \ell_{r} A_{r}$ where each $\ell_{i}, i \leq r$, is at position $k_{i}$, and such that the concatenated block $A_{1} A_{2} \ldots A_{r}$ consists of the increasing sequence of values in $[n] \backslash\left\{\ell_{1}, \ell_{2}, \ldots, \ell_{r}\right\}$. Therefore, $\ell_{i+1}$ is greater than all elements in $A_{i}$ which means that the set of left-to-right maxima of $\pi$ is $L(\pi)=\left\{k_{1}, k_{2}, \ldots, k_{r}\right\}$ with $\pi\left(k_{i}\right)=\ell_{i}$ for $i \leq r$. By construction, $\pi$ avoids the pattern 321 and $P$ is its associated Dyck path which gives a bijection from $S_{n}^{\sim_{\ell}^{\ell}}$ to $\mathcal{P}_{n}$ that induces Theorem 1. In the following, the permutation $\pi$ will be called the associated permutation of $P$. Notice that a similar construction already exists in the literature (see the Krattenthaler bijection $\Psi$ defined in [9], Section 4).

THEOREm 2.1 The sets $S_{n}^{\sim_{\ell}}$ (resp. $S_{n}(321)^{\left.\sim_{\ell}\right)}$ ), $n \geq 1$, are enumerated by the Catalan numbers (sequence A000108 in the on-line Encyclopedia of Integer Sequences [18]).

\subsection{Equivalence classes for classical subsets of permutations}

In this part we give several enumerating results for classical subsets of $S_{n}$ (see Table 1).

Theorem 2.2 The sets $D_{n}^{\sim \ell}, n \geq 1$, are enumerated by the Fine numbers (A000108 in [18]).

Proof. Using the above construction, we construct a Dyck path $P$ of length $2 n$ from $\pi \in D_{n}$. Since $\pi$ does not contain any fixed point, $P$ does not contain any peak of height one. Conversely, let $P$ be a Dyck path with no peak of height one and $\pi \in S_{n}(321)$ be its associated permutation of $P$ (see the above construction). Since $P$ does not contain any peak of height one, this implies that there does not exist $i \in L(\pi)$ such that $\pi_{i}=i$. Now, for a contradiction, let us assume that there is $j \notin L(\pi)$ such that $\pi_{j}=j$. Since $j \notin L(\pi)$, there is $i<j$ such that $\pi_{i}>j=\pi_{j}$. So, there are at most $j-2$ values $\pi_{k}<\pi_{j}$ for $k<j$, or equivalently there is at least one value $\pi_{k}<\pi_{j}$ for $k>j$ which contradicts the fact that $\pi$ avoids the pattern 321. Finally, the result follows because the set of Dyck paths with no peak of height one is enumerated by the Fine numbers (see [4]).

THEOREM 2.3 Let $\operatorname{Irr}_{n}$ be the set of permutations $\pi \in S_{n}$ such that $\pi_{i} \neq \pi_{i+1}-1,1 \leq i \leq n-1$. The sets $\operatorname{Irr}_{n}^{\sim \ell}, n \geq 1$, are enumerated by the sequence A078481 in [18]. 
Proof. Let $\pi$ be a permutation in $\operatorname{Irr}_{n}$. Then the Dyck path associated with $\pi$ does not contain any consecutive steps of the form $U D U D$. Conversely, let $P$ be a Dyck path which does not contain any occurence of consecutive $U D U D$, and $\pi \in S_{n}(321)$ be its associated permutation. Since $P$ does not contain any occurrence of $U D U D$, this implies that there does not exist $i \in L(\pi)$ such that $\pi_{i}=\pi_{i+1}-1$. In the case where there is $j, j \notin L(\pi)$ such that $\pi_{j}=\pi_{j+1}-1$, we define the blocks of maximal length $J_{1}, J_{2}, \ldots, J_{s}$ of the form $a, a+1, \ldots, b$ such that $a \leq b$ and $\pi_{a}=\pi_{a+1}-1, \pi_{a+1}=\pi_{a+2}-1, \ldots$, $\pi_{b-1}=\pi_{b}-1$ where $a \notin L(\pi)$. We consider the permutation $\sigma$ obtained from $\pi$ by the following process: for each block $J_{k}=a, a+1, \ldots, b, 1 \leq k \leq s$, in the one-line notation of $\pi$ we replace the block $\pi\left(J_{k}\right)$ with its mirror $\pi\left(J_{k}^{\prime}\right)$ where $J_{k}^{\prime}=b, b-1, \ldots, a$. So, $\sigma$ and $\pi$ belong to the same class, and $\sigma \in I r r_{n}$. For instance, the Dyck path $U U U U D D D D$ would produce $\pi=4123 \notin I r r_{4}$, then applying the described process, the permutation $\sigma=4321 \in \mathrm{Irr}_{4}$ is obtained. Finally, the result is obtained since the set of Dyck paths with no occurrence of $U D U D$ is enumerated by the sequence A078481 in [18] (see [17]).

Theorem 2.4 The sets $I_{n}^{\sim}, n \geq 1$, are enumerated by the Motzkin numbers (A001006 in [18]).

Proof. We will show that each equivalence class contains a unique involution that avoids the pattern 4321 (see A001006 in [18] and [6] for the enumeration of $I_{n}(4321)$ by Motzkin numbers). Let $\pi$ be an involution in $I_{n}$. If there exists a position $i, i<\pi_{i}$, such that $i$ is not a left-to-right maximum, then there is $j \in[n]$ such that $j<i<\pi_{i}<\pi_{j}$ which means that $\pi$ contains the pattern 4321. So, we define the involution $\sigma$ satisfying $L(\pi)=L(\sigma)$ and verifying the additional conditions $\sigma_{i}=i$ whenever $i \notin L(\pi)$. By construction, $\sigma$ avoids the pattern 4321 and belongs to the same class of $\pi$. Conversely, let $\sigma$ be an involution avoiding the pattern 4321. Then, the inequality $j<i<\sigma_{i}<\sigma_{j}, i, j \in[n]$ does not occur. Therefore, if $i \notin L(\sigma)$, then $i$ is necessarily a fixed point. Therefore, there is a unique involution $\sigma \in I_{n}(4321)$ having $L(\sigma)$ as set of left-to-right maxima.

TheORem 2.5 The sets $C_{n}^{\sim}, n \geq 1$, are enumerated by the Catalan numbers (A000108 in [18]).

Proof. Any permutation $\pi \in S_{n-1}$ can uniquely be decomposed as a product of transpositions

$$
\pi=\left\langle p_{1}, 1\right\rangle \cdot\left\langle p_{2}, 2\right\rangle \cdots\left\langle p_{n-1}, n-1\right\rangle
$$

where $p_{i}$ are some integers such that $1 \leq p_{i} \leq i \leq n-1$ (see for instance [1]).

Let $\phi$ be the map from $S_{n-1}$ to $S_{n}$ defined, for every $\pi \in S_{n-1}$, by

$$
\phi(\pi)=\langle 1,1\rangle \cdot\left\langle p_{1}, 2\right\rangle \cdots\left\langle p_{n-1}, n\right\rangle
$$

where $\pi=\left\langle p_{1}, 1\right\rangle \cdot\left\langle p_{2}, 2\right\rangle \cdots\left\langle p_{n-1}, n-1\right\rangle$.

Using Corollary 1 in [1], $\phi$ is a bijection from $S_{n-1}$ to $C_{n}$ satisfying $L(\pi)=L(\phi(\pi))$ for any $\pi \in S_{n}$ and such that $\phi(\pi)(k)=\pi(k)+1$ for $k \in L(\pi)$. Therefore, $\phi$ induces a bijection from $S_{n-1}^{\sim \ell}$ to $C_{n}^{\sim \ell}$. With Theorem 2.1, the cardinality of $C_{n}^{\sim_{\ell}}$ is the $(n-1)$-th Catalan number.

\subsection{Equivalence classes for $S_{n}(\alpha)^{\sim \ell}$ with $\alpha \in S_{3}$}

In this part we give several enumerating results for the sets $S_{n}(\alpha)^{\sim} \sim_{\ell}$ where the pattern $\alpha$ lies in $S_{3}$ (see Table 2). 


\begin{tabular}{|c|c|c|c|}
\hline Set & Sequence & Sloane & $a_{n}, 1 \leq n \leq 9$ \\
\hline$S_{n}^{\sim_{\ell}}$ & Catalan & $A 000108$ & $1,2,5,14,42,132,429,1430,4862$ \\
\hline$C_{n}^{\sim_{\ell}}$ & Catalan & $A 000108$ & $1,1,2,5,14,42,132,429,1430$ \\
\hline$I_{n}^{\sim^{\ell}}$ & Motzkin & $A 001006$ & $1,2,4,9,21,51,127,323,835$ \\
\hline$D_{n}^{\sim_{\ell}}$ & Fine & $A 000957$ & $0,1,2,6,18,57,186,622,2120$ \\
\hline$I r r_{n}^{\sim_{\ell}}$ & Dyck with no UDUD & $A 078481$ & $1,1,3,7,19,53,153,453,1367$ \\
\hline
\end{tabular}

Table 1: Number of equivalence classes for classical subsets of permutations.

Theorem 1 proves that $S_{n}(321)^{\sim_{\ell}}$ is enumerated by the $n$th Catalan number. Let $\phi$ be the bijection from $S_{n}(321)$ to $S_{n}(312)$ described (modulo a basic symmetry) in [3] (Lemma 4.3, page 148). It has the property to leave all left-to-right maxima fixed. Therefore, it induces a bijection from $S_{n}(321)^{\sim}$ to $S_{n}(312)^{\sim \ell}$.

Now, let us examine the cases where the pattern $\alpha$ belongs to $\{123,132,213,231\}$.

THEOREM 2.6 The sets $S_{n}(123)^{\sim_{\ell}}, n \geq 1$, are enumerated by the central polygonal numbers $1+\frac{n(n-1)}{2}$ (A000124 in [18]).

Proof. Let $\pi$ be a permutation in $S_{n}(123)$. It is straightforward to see that the left-to-right maxima of $\pi$ are 1 and $i$ where $\pi_{i}=n$ for some $i, 1 \leq i \leq n$. We necessarily have $i-1 \leq \pi_{1}$ because the condition $\pi_{1}<\pi_{j}<n$ implies $j<i$. Since the values $i$ and $j, 1 \leq i, j \leq n$, characterize a class in $S_{n}(123)^{\sim \ell}$, it follows that the cardinality of $S_{n}(123)^{\sim_{\ell}}$ is given by $1+\sum_{i=2}^{n} \sum_{j=i-1}^{n-1} 1=1+\frac{n(n-1)}{2}$.

TheOREm 2.7 For $\alpha \in\{132,213,231\}$, the sets $S_{n}(\alpha)^{\sim}, n \geq 1$, are enumerated by the binary numbers $2^{n-1}$.

Proof. Let $\pi$ be a permutation in $S_{n}(231)$. It can be written $\pi=\sigma n \gamma$ where $\sigma \in S_{k}(231)$ for some $k, 0 \leq k \leq n-1$, and $\gamma$ is obtained from a permutation in $S_{n-k-1}(231)$ by adding $k$ on all these entries. Therefore, the set $L(\pi)$ of left-to-right maxima of $\pi$ is the union of $\{k+1\}$ with the set $L(\sigma)$ of left-to-right maxima of $\sigma$. For $n \geq 1$, let $a_{n}$ be the cardinality of $S_{n}(231)^{\sim_{\ell}}$. Varying $k$ from 0 to $n-1$, we have $a_{n}=1+\sum_{k=1}^{n-1} a_{k}$ anchored with $a_{1}=1$. Thus, we deduce $a_{n}=2^{n-1}$ for $n \geq 1$.

Basic symmetries on permutations allow to obtain the result whenever $\alpha$ lies in $\{132,312\}$.

\section{Enumeration of classes under $d$-equivalence relation}

In this section two permutations $\pi$ and $\sigma$ belong to a same class whenever $D D(\pi)=D D(\sigma)$, i.e., if the set of pairs $\left(\pi_{i}, \pi_{i+1}\right)$ for $i \in D(\pi)$ is equal to the set of pairs $\left(\sigma_{i}, \sigma_{i+1}\right)$ for $i \in D(\sigma)$.

A partition $\Pi$ of $[n]$ is any collection of non-empty pairwise disjoint subsets, called blocks, whose union is $[n]$. The standard form of $\Pi$ is $\Pi=B_{1} / B_{2} / \ldots$, where the blocks $B_{i}$ are arranged so that their smallest elements are in increasing order. For convenience, we assume also that elements in a 


\begin{tabular}{|c|c|c|c|}
\hline Pattern & Sequence & Sloane & $a_{n}, 1 \leq n \leq 9$ \\
\hline$\{123\}$ & Central polygonal & $A 000124$ & $1,2,4,7,11,16,22,29,37$ \\
\hline$\{312\},\{321\}$ & Catalan & $A 000108$ & $1,2,5,14,42,132,429,1430,4862$ \\
\hline$\{132\},\{213\},\{231\}$ & Binary & $A 000079$ & $1,2,4,8,16,32,64,128,256$ \\
\hline
\end{tabular}

Table 2: Number of equivalence classes for permutations avoiding one pattern in $\mathcal{S}_{3}$.

same block are arranged in decreasing order. From a permutation $\pi \in S_{n}$, we associate the unique partition $\Pi$ defined as follows. Two elements $x>y$ belong to the same block in $\Pi$ if and only if there exist $i$ and $j, i<j$, such that $\pi_{i}=x>\pi_{i+1}>\cdots>\pi_{j-1}>\pi_{j}=y$. Conversely, any partition $\Pi=B_{1} / B_{2} / \ldots / B_{k}, k \geq 1$, is the associated to the permutation $B_{1} B_{2} \ldots B_{k}$. Theorem 8 becomes a straightforward consequence.

TheOREM 3.1 The sets $S_{n}^{\sim d}, n \geq 1$, are enumerated by the Bell numbers (A000110 in [18]).

Theorem 3.2 The sets $S_{n}(321)^{\sim d}, n \geq 1$, are enumerated by the Motzkin numbers (A001006 in [18]).

Proof. Let $\pi \in S_{n}(321)$ and $D D(\pi)=\left\{\left(M_{1}, m_{1}\right),\left(M_{2}, m_{2}\right), \ldots,\left(M_{r}, m_{r}\right)\right\}, r \geq 0$, be the set of pairs $\left(\pi_{i}, \pi_{i+1}\right)$ where $i$ is a descent of $\pi$. Since $\pi$ avoids $321, D D(\pi)$ does not contain two pairs of the form $\left(\pi_{i}, \pi_{i+1}\right)$ and $\left(\pi_{i+1}, \pi_{i+2}\right)$. Then, we define the involution $\sigma \in I_{n}$ as follows: $\sigma\left(M_{i}\right)=m_{i}, \sigma\left(m_{i}\right)=M_{i}$ for $1 \leq i \leq r$ and $\sigma(k)=k$ if $k$ does not appear in any pair of $D D(\pi)$. For a contradiction, let us assume that $\sigma$ contains a pattern 4321 . Then there exist two pairs $\left(M_{i}, m_{i}\right)$ and $\left(M_{j}, m_{j}\right)$ in $D D(\pi)$ such that $M_{i}>M_{j}>m_{j}>m_{i}$. If the descent $\left(M_{i}, m_{i}\right)$ is on the left (in $\left.\pi\right)$ of the descent $\left(M_{j}, m_{j}\right)$, then the subsequence $M_{i} M_{j} m_{j}$ is a pattern 321 of $\pi$; otherwise, the subsequence $M_{j} m_{j} m_{i}$ also is a 321-pattern. In the two cases we obtain a contradiction, which ensures that the involution $\sigma$ avoids the pattern 4321.

Conversely, let $\sigma$ be an involution avoiding the pattern 4321. There exists a sequence of pairs $\left(M_{1}, m_{1}\right), \ldots,\left(M_{r}, m_{r}\right), r \geq 0$, such that $M_{i}<M_{i+1}, m_{i}<m_{i+1}$ for $i \leq r-1$ and such that $M_{i}>m_{i}$, $\sigma\left(M_{i}\right)=m_{i}$ and $\sigma\left(m_{i}\right)=M_{i}$ for $i \leq r$ and $\sigma(k)=k$ for $k \in[n] \backslash\left\{M_{1}, \ldots, M_{r}, m_{1}, \ldots, m_{r}\right\}$. We define the permutation $\pi$ with the following process. We start with the sequence $M_{1} m_{1} M_{2} m_{2} \ldots M_{r} m_{r}$; we insert in increasing order all other values $k$ satisfying $\sigma(k)=k$ as follows: if $m_{i-1}<k<m_{i}$ then we insert $k$ between $m_{i-1}$ and $M_{i}$; if $k<m_{1}$ then we insert $k$ before $M_{1}$; and if $k \geq m_{r}$ then we insert $k$ after $m_{r}$. Obviously, this construction induces that $\pi$ avoids 321. Moreover, $\sigma$ can be obtained from $\pi$ by the construction of the beginning of this proof. Thus, there is a bijection between $S_{n}(321)^{\sim}$ and $I_{n}(4321)$ which is enumerated by the Motzkin numbers (see A001006 in [18] and [6]).

Lemma 3.3 Let $\pi$ and $\pi^{\prime}$ be two permutations in $S_{n}(132)$ belonging to the same d-equivalence class. If we have $\pi_{1}=\pi_{1}^{\prime}$ then $\pi=\pi^{\prime}$.

Proof. We proceed by induction on $n$. A simple observation gives the result for $n \leq 3$. Now, let us assume that Lemma 1 is true for $k \leq n-1$. Let $\pi$ and $\pi^{\prime}$ be two permutations in $S_{n}(132)$ such that $\pi_{1}=\pi_{1}^{\prime}$. We can write $\pi=\alpha n \beta$ (resp. $\pi^{\prime}=\alpha^{\prime} n \beta^{\prime}$ ) where $\beta \in S_{k}(132)$ for some $k, 0 \leq k \leq n-1$ (resp. $\beta^{\prime} \in S_{k^{\prime}}(132)$ for some $k^{\prime}, 0 \leq k^{\prime} \leq n-1$ ), and $\alpha$ (resp. $\alpha^{\prime}$ ) is obtained from a permutation in 
$S_{n-k-1}(132)$ (resp. $\left.S_{n-k^{\prime}-1}(132)\right)$ by adding $k$ (resp. $k^{\prime}$ ) on all these entries. Let $m$ (resp. $m^{\prime}$ ) be the minimal value of $\alpha$ (resp. $\alpha^{\prime}$ ).

Without loss of generality, we assume that $m^{\prime} \leq m \leq \pi_{1}=\pi_{1}^{\prime}$. For a contradiction, assume that $m^{\prime}<m$. So, there is two consecutive entries $a$ and $m^{\prime}$ in $\alpha^{\prime}$ such that $a>m^{\prime}$. As $m^{\prime}<m$, the descent $\left(a, m^{\prime}\right)$ does not appear in $\alpha$. Thus, $\left(a, m^{\prime}\right)$ appears in $\beta$. Let $\alpha_{1}$ be the first value of $\alpha$; the subsequence $\alpha_{1} a m^{\prime}$ is necessarily a pattern 132 which is a contradiction. Thus, we have $m=m^{\prime}$ and then $k=k^{\prime}$. We deduce $\alpha$ and $\alpha^{\prime}$ (resp. $\beta$ and $\beta^{\prime}$ ) are $d$-equivalent and the recurrence hypothesis gives $\alpha=\alpha^{\prime}$. Moreover the descent $\left(n, \beta_{1}\right)$ is equal to the descent $\left(n, \beta_{1}^{\prime}\right)$ and then $\beta_{1}=\beta_{1}^{\prime}$. Using the recurrence hypothesis we conclude $\beta=\beta^{\prime}$ and then, $\pi=\pi^{\prime}$.

THEOREM 3.4 The sets $S_{n}(132)^{\sim}, n \geq 1$, are enumerated by $c_{n}-c_{n-1}+1$ where $c_{n}=\frac{1}{n+1}\left(\begin{array}{c}2 n \\ n\end{array}\right)$ is the $n$-th Catalan number.

Proof. Let $a_{n}$ be the cardinality of $S_{n}(132) \sim_{d}$. We distinguish three kinds of classes: (1) classes with a representative $\pi$ satisfying $\pi_{1}=n$; (2) classes with a representative $\pi$ satisfying $\pi_{n}=n$; (3) the remaining classes.

Case (1). Such a class contains a permutation $\pi$ such that $\pi_{1}=n$, i.e., $\pi=n \pi^{\prime}$ with $\pi^{\prime} \in S_{n-1}(132)$. Using Lemma 3.3 , there is a unique $\sigma=\pi^{\prime} \in S_{n-1}(132)$ such that $n \sigma$ and $\pi$ belong to the same class. Thus, the number of classes in this case is also the cardinality of $S_{n-1}(132)$, that is the $(n-1)$-th Catalan number $c_{n-1}$.

Case (2). Such a class contains a permutation $\pi$ such that $\pi_{n}=n$. So, the number of classes in this case is also the number of elements in $S_{n-1}(132)^{\sim_{d}}$, that is $a_{n-1}$.

Case (3). Now we consider the classes that do not lie in the two previous cases. Any permutation $\pi$ of such a class satisfies $\pi_{i}=n$ for some $i \in[2, n-1]$, and since $\pi$ avoids $132, \pi$ can be written $\pi=\alpha n \beta$ where $\beta \in S_{n-i}(132)$ and $\alpha$ is obtained by adding $(n-i)$ on all entries of a permutation in $S_{i-1}(132)$.

Let us consider $j, 1 \leq j \leq i-1$, the position where $\alpha$ reaches its minimum $m$.

If $j=1$ then $\pi=(n-i+1) \ldots(n-1) n \beta$ and this permutation lies in the same class of $n \beta(n-$ $i+1) \ldots(n-1)$ that satisfies Case (1). Then, $j=1$ does not occur.

Now we assume $j \geq 2$ and let $\sigma$ be a permutation in $S_{n}(132)$ lying in the same class of $\pi$. Then, $\sigma$ must contain the two descents $\left(n, \pi_{i+1}\right)$ and $\left(\pi_{j-1}, m\right)$. These two descents necessarily appear in the same order as in $\pi$ (otherwise, a pattern 132 would be created with $\pi_{i+1} \pi_{j-1} m$ ). Thus, the minimum $m^{\prime}$ of values on the left of $n$ in $\sigma$ is necessarily less or equal to $m$. For a contradiction, let us assume that $m^{\prime}<m$. The value $m^{\prime}$ appears necessarily on the right of the descent $\left(\pi_{j-1}, m\right)$ in $\sigma$ (otherwise, $m^{\prime} \pi_{j-1} m$ would be a pattern 132). Therefore, a descent of the form $(a, b), a \geq m$ and $b<m$ would necessarily exists in $\sigma$, which is not possible because such a descent cannot belong in $\pi$.

Thus, we deduce $m=m^{\prime}$ and $\sigma$ has the similar decomposition $\sigma=\alpha^{\prime} n \beta^{\prime}$ where $\beta^{\prime} \in S_{n-i}(132)$ and $\alpha^{\prime}$ is obtained by adding $(n-i)$ on all entries of some permutation in $S_{i-1}(132)$. So, $\alpha$ (resp. $\beta$ ) is equivalent to $\alpha^{\prime}$ (resp. $\beta^{\prime}$ ). Hence, Lemma 3.3 implies that $\beta=\beta^{\prime}$. Then, for a given $i \in[2, n-1]$, there are exactly $c_{n-i} \cdot\left(a_{i-1}-1\right)$ classes verifying this case (we subtract one to $a_{i-1}$ because we do not consider $\pi=(n-i+1) \ldots n \beta)$.

So, such classes are enumerated by $\sum_{k=2}^{n-1}\left(a_{k-1}-1\right) \cdot c_{n-k}$. 
Considering the three cases, the cardinality $a_{n}$ of $S_{n}(132)^{\sim_{d}}$ satisfies for $n \geq 2$,

$$
a_{n}=c_{n-1}+a_{n-1}+\sum_{k=2}^{n-1}\left(a_{k-1}-1\right) \cdot c_{n-k} .
$$

A simple calculation proves that $a_{n}=c_{n}-c_{n-1}+1$ for $n \geq 2$.

THeOREM 3.5 The sets $S_{n}(123)^{\sim_{d}}, n \geq 2$, are enumerated by $c_{n}+n-(n+2) \cdot 2^{n-3}+\frac{(n-2)(n-1)}{2}$ where $c_{n}=\frac{1}{n+1}\left(\begin{array}{c}2 n \\ n\end{array}\right)$ is the $n$-th Catalan number.

Proof. Let $\pi$ be a permutation in $S_{n}(123)$. Then $\pi$ has a unique decomposition into blocks of decreasing sequences, i.e., $\pi=A_{1} A_{2} \ldots A_{r}, 1 \leq i \leq r$, where blocks $A_{i}$ consist of sequences of decreasing values (possibly reduced to one value) and such that $\ell_{i}<f_{i+1}$ for $1 \leq i \leq r-1$, where $f_{i}$ (resp. $\ell_{i}$ ) is the first (resp. last) element of $A_{i}$.

We distinguish three cases (1) $r=1$; (2) $r=2$; and (3) $r \geq 3$.

Case (1). We necessarily have $\pi=n(n-1) \ldots 21$ and its equivalence class contains only one element. Case (2). We have $\pi=A_{1} A_{2}$ with $\ell_{1}<f_{2}$. (i) If $f_{1}<\ell_{2}$ then the class of $\pi$ contains only one element since the permutation $\sigma=A_{2} A_{1}$ is not there. Therefore, there are $(n-1)$ such classes. (ii) If $f_{1}>\ell_{2}$ then the class of $\pi$ contains exactly two elements $\pi$ and $\sigma=A_{2} A_{1}$. Since the number of permutations of length $n$ with $n-2$ descents is $2^{n}-(n+1)$, the number of classes for the subcase $(i i)$ is $\frac{2^{n}-(n+1)-(n-1)}{2}=2^{n-1}-n$. Finally, there are $2^{n-1}-n+n-1=2^{n-1}-1$ classes for Case (2).

Case (3). $\pi$ contains at least three blocks. We decompose $\pi=A_{1} B A_{r}$ where $B=A_{2} \ldots A_{r-1}, r \geq 3$, such that $f_{2}=n, \ell_{r-1}=1$ with $A_{1}$ and $A_{r}$ possibly empty.

Let $\sigma$ be a permutation in $S_{n}(123)$ belonging to the class of $\pi$. We will prove that $\sigma$ is either $\pi$ or $A_{r} B A_{1}$.

For this, we will prove that the block $B$ also appears in $\sigma$. It is obvious whenever $B$ is a decreasing sequence. Now, let us assume that $B$ is the concatenation of at least two blocks, that is $B=A_{2} \ldots A_{r-1}$ with $r \geq 4$.

(i) For a contradiction, we suppose that there exist $i$ and $j, 2 \leq i<j \leq r-1$ such that $A_{i}$ appears on the right of $A_{j}$ into $\sigma$, i.e., $\sigma=\alpha A_{j} \beta A_{i} \gamma$ for some $\alpha, \beta, \gamma$ possibly empty. Since $\sigma$ avoids 123 and $f_{j}<f_{i}, \alpha$ does not contain any value $a$ such that $a<f_{j}$ (otherwise $a f_{j} f_{i}$ would be a pattern 123). Also, $\alpha$ does not contain any value $a$ such that $a>f_{j}$ (otherwise there would be $b \leq a$ such that $\left(b, f_{j}\right)$ is descent in $\sigma$ that does not appear in $\pi$ ). Thus $\alpha$ is necessarily empty. By a simple symmetry, $\gamma$ is also empty. This implies that all other blocks of $\pi$ appear between the two blocks $A_{j}$ and $A_{i}$ in $\sigma$. Thus, all other blocks consist of values $a$ such that $a \in\left[1, \ell_{j}-1\right] \cup\left[f_{i}+1, n\right]$ (otherwise $\ell_{j} a f_{i}$ would be a pattern 123). Since $\pi$ contains at least three blocks there is at least one block between $A_{j}$ and $A_{i}$ in $\sigma$.

The case $\ell_{j}=1$ does not occur. Indeed, this would mean that all blocks between $A_{j}$ and $A_{i}$ contain values $x$ greater than $f_{i}$ which creates a descent of the form $\left(x, f_{i}\right)$ that does not appear in $\pi$. A similar argument proves that $f_{i}=n$ does not occur.

Let us consider the case where $n$ and 1 do not appear in $A_{i}$ and $A_{j}$. Since $B$ contains at least two blocks, $n$ and 1 do not appear in the same block in $B$. Let $R$ (resp. $S$ ) be the block containing $n$ (resp. 1). In $\sigma$, the last element $\ell(R)$ of $R$ is necessarily less than $\ell_{j}$ (otherwise $\sigma$ would contain a pattern 123 , that is $\ell_{j} \ell(R) x$ where $x$ is the value just after $\ell(R)$ in $\left.\sigma\right)$. A same argument shows that the first 
element $f(S)$ of $S$ is greater than $f_{i}$. In $\pi$, this would mean that $\ell(R) \ell_{i} f(S)$ is a pattern 123 which is a contradiction. Finally, all blocks of $B$ appear in $\sigma$ in the same order as $\pi$.

(ii) Now we will prove that $A_{1}$ does not appear between $A_{2}$ and $A_{r-1}$ in $\sigma$. For a contradiction, let us assume that $A_{1}$ appears between $A_{2}$ and $A_{r-1}$ in $\sigma$. Let $a$ be the value of $\sigma$ just after the block $A_{1}$. If $a<\ell_{1}$ then $\sigma$ contains the descent $\left(\ell_{1}, a\right)$ that does not appear in $\pi$; otherwise, $\ell_{2} \ell_{1} a$ would be a pattern 123 in $\sigma$ which gives a contradiction. A same argument proves that $A_{r}$ does not appear between $A_{2}$ and $A_{r-1}$ in $\sigma$.

Therefore, we deduce that either $\sigma=\pi$ or $\sigma=A_{r} B A_{1}$.

Now we will enumerate permutations $\pi \in S_{n}(123)$ of Case (3) such that there is $\sigma \in S_{n}(123)$, $\sigma \neq \pi$, belonging to the same class of $\pi$, i.e., $A_{1} B A_{r}$ and $A_{r} B A_{1}$ do not contain any pattern 123 . This case is characterized by the fact that there is no value $a$ in the block $B$ such that $\min \left\{\ell_{1}, \ell_{r}\right\}<a<$ $\max \left\{f_{1}, f_{r}\right\}$ (otherwise, one of the two permutations $A_{1} B A_{r}, A_{r} B A_{1}$ would contain the pattern 123). See Figure 1 for a graphical representation of such a permutation.

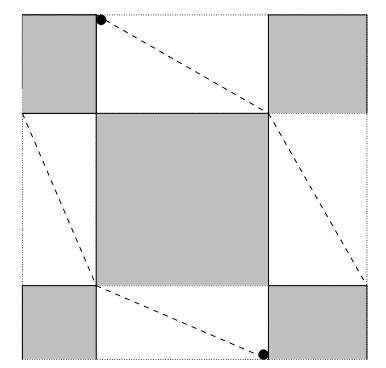

Figure 2: Illustration of $\pi=A_{1} B A_{r} \in S_{n}(123)$ having two elements in its class.

If $B$ contains only one block, $A_{1}$ and $A_{r}$ are non-empty blocks. Varying the size $k$ of $B$ from 2 to $n-2$, and the size $\ell$ of $A_{1}$ from 1 to $n-k-1$, the number of permutations having two elements in its class is $a_{n}=\sum_{k=2}^{n-2}(k-1) \cdot \sum_{\ell=1}^{n-k-1}\left(\begin{array}{c}n-k \\ \ell\end{array}\right)$.

If $B$ contains at least two blocks, $A_{1}$ and $A_{r}$ are blocks (possibly empty). Varying the size $k$ of $B$ from 4 to $n-1$ and varying the size $\ell$ of $A_{1}$ from 0 to $n-k$, the number of permutations having two elements in its class is $b_{n}=\sum_{k=4}^{n-1}\left(2^{k-2}-(k-1)\right) \cdot \sum_{\ell=0}^{n-k}\left(\begin{array}{c}n-k \\ \ell\end{array}\right)$.

Finally, the number of classes in $S_{n}(123)$ is obtained from $c_{n}$ by subtracting the number of classes having two elements, that is $c_{n}-\frac{1}{2}\left(2^{n}-2 n+a_{n}+b_{n}\right)=c_{n}-(n+2) \cdot 2^{n-3}+\frac{n(n-1)}{2}+1$.

\section{References}

[1] J.-L. BARIL, Statistics-preserving bijections between classical and cyclic permutations, Inform. Process. Lett., 113 (2003) 17-22.

[2] J.-L. Baril, T. Mansour and A. Petrossian, Equivalence classes of permutations modulo excedances, J. Comb., 5 (2014) 453-469. 


\begin{tabular}{|c|c|c|c|}
\hline Pattern & Sequence & Sloane & $a_{n}, 1 \leq n \leq 9$ \\
\hline\{\} & Bell & $A 000110$ & $1,2,5,15,52,203,877,4140,21147$ \\
\hline$\{231\},\{312\}$ & Catalan & $A 000108$ & $1,2,5,14,42,132,429,1430,4862$ \\
\hline$\{321\}$ & Motzkin & $A 001006$ & $1,2,4,9,21,51,127,323,835$ \\
\hline$\{132\},\{213\}$ & $c_{n}-c_{n-1}+1$ & New & $1,2,4,10,29,91,298,1002,3433$ \\
\hline$\{123\}$ & $c_{n}-(n+2) \cdot 2^{n-3}+\frac{n(n-1)}{2}+1$ & New & $1,2,4,9,25,84,307,1139,4195$ \\
\hline
\end{tabular}

Table 3: Number of equivalence classes for permutations avoiding at most one pattern of $\mathcal{S}_{3}$.

[3] M. Bona, Combinatorics of permutations, Chapman and Hall/CRC, Second Edition, 2012.

[4] E. Deutsch and L. Shapiro, A survey of the Fine numbers, Discrete Math., 241 (2001) $241-265$.

[5] D. C. Foata and M. P. Schützenberger, Théorie Géométrique des Polynômes Euleriens, Lecture Notes in Math., 138, Springer-Verlag, Berlin, 1970.

[6] O. Guibert, E. Pergola And R. Pinzani, Vexillary involutions are enumerated by Motzkin numbers, Ann. Comb., 5 (2001) 153-174.

[7] S. Kitaev, Patterns in permutations and words, Springer-Verlag, 2011.

[8] D. E. KNuth, The art of computer programming, Volume I, Addison-Wesley, Reading MA, Third edition, 1997.

[9] C. Krattenthaler, Permutations with restricted patterns and Dyck paths, Adv. in Appl. Math., 27 (2001) 510-530.

[10] T. Mansour, Combinatorics of Set Partitions, Discrete Mathematics and its Applications, Chapman \& Hall/CRC, 2013.

[11] T. Mansour, Permutations avoiding a pattern from $S_{k}$ and at least two patterns from $S_{3}$, Ars. Combin., 62 (2002) 227-239.

[12] T. Mansour and A. Vainshtein, Restricted 132-avoiding permutations, Adv. Appl. Math., 26 (2001) 258-269.

[13] R. Simion And F.W. Schmidt, Restricted permutations, European J. Combin., 6 (1985) $383-406$.

[14] J. West, Permutations with forbidden subsequences and stack-sortable permutations, Ph.D. Thesis, MIT, 1990.

[15] J. West, Generating trees and forbidden subsequences, Local Proceedings of FPSAC 1994, $441-450$. 
[16] J. West, Generating trees and the Catalan and Schröder numbers, Discrete Math., 146 (1995) 247-262.

[17] A. Sapounakis, I. Tasoulas and P. Tsikouras, Counting strings in Dyck paths, Discrete Math., 307 (2007) 2909-2924.

[18] N.J.A. Sloane, On-Line Encyclopedia of Integer Sequences, http://oeis.org/.

[19] R. P. Stanley, Enumerative Combinatorics, Volume I, Cambridge University Press, 2002. 